\title{
Review Article \\ Prognostic Value of Galectin-3 in Patients with Heart Failure
}

\author{
Ivica Bošnjak, ${ }^{1}$ Kristina Selthofer-Relatić, ${ }^{1,2}$ and Aleksandar Včev ${ }^{2,3}$ \\ ${ }^{1}$ Department for Cardiovascular Disease, Clinic for Internal Medicine, University Hospital Centre Osijek, \\ J. Huttlera 4, 31000 Osijek, Croatia \\ ${ }^{2}$ Faculty of Medicine Osijek, University Josipi Juraj Strossmayer Osijek, J. Huttlera 4, 31000 Osijek, Croatia \\ ${ }^{3}$ Department for Gastroenterology, Clinic for Internal Medicine, University Hospital Centre Osijek, Osijek, Croatia \\ Correspondence should be addressed to Ivica Bošnjak; dribosnjak79@gmail.com
}

Received 9 October 2014; Revised 5 March 2015; Accepted 6 March 2015

Academic Editor: Allan Jaffe

Copyright (c) 2015 Ivica Bošnjak et al. This is an open access article distributed under the Creative Commons Attribution License, which permits unrestricted use, distribution, and reproduction in any medium, provided the original work is properly cited.

Galectins are a family of soluble $\beta$-galactoside-binding lectins that have important role in inflammation, immunity, and cancer. Galectin-3 as a part of this lectin family plays a very important role in development of heart failure. According to recent papers, galectin-3 plasma level correlates with heart failure outcome, primarily with rehospitalisation and death from heart failure. This paper summarizes the most recent advances in galectin-3 research, with the accent on the role of galectin-3 in pathophysiology of myocardial remodelling and heart failure development-with preserved and reduced ejection fraction, and some implication on development of new disease modifying drugs.

\section{Introduction}

Heart failure (HF) can be defined as a complex mechanical and neurohumoral syndrome manifested by hemodynamic congestion presenting with dyspnea, orthopnea, paroxysmal nocturnal dyspnea, or peripheral oedema, along with the presence of objective evidence of cardiac dysfunction [1, 2]. A new symptom in heart failure, bendopnea, has been described recently. Bendopnea is mediated via a further increase in filling pressures during bending when filling pressures are already high, particularly if cardiac index is reduced [3]. Heart failure remains one of the most prevalent and challenging medical conditions. Despite advances in treatment, morbidity and mortality in $\mathrm{HF}$ are very high, thus representing one of the most costly medical conditions [4]. Until now, HF has been considered to be associated with impaired cardiac contractility and cardiac dilation, but it has become evident that a considerable portion of patients presenting with clinical HF have a normal ejection fraction (EF). Now we can distinguish heart failure with reduced ejection fraction $<40 \%$ (HFrEF) and heart failure with preserved ejection fraction $>40 \%$ (HFpEF) $[5,6]$. The pathophysiology concept of HF is related to the concept of myocardial remodelling. Increased stress or injury to the myocardium due to hypertension, diabetes mellitus, or ongoing ischemia can contribute to cardiac remodelling [7]. Response to acute or chronic damage can involve activation of immune cells to the myocardium, production of cell signaling proteins from local pericytes, mast cells, and macrophages, resulting in activation of resident fibroblasts and myofibroblasts, and deposition of collagen into extracellular matrix, which is correlated with collagen generating cardiac fibrosis [8]. There are many regulators involved in the pathophysiology of cardiac fibrosis. One of them is galectin-3.

\section{Galectin-3 and Myocardial Remodelling}

Galectin-3 (Gal-3) is a member of a galectin family involved in numerous physiological and pathological processes, some of which, inflammation and fibrosis, are pivotal contributing pathophysiological mechanisms to the development of HF. Gal-3 is a $29-35 \mathrm{kDa}$ chimaera-type galectin which is unique in that it is the only member of the galectin family with an extended $\mathrm{N}$-terminal domain constituted of tandem repeats of short amino acid segments linked to a single C-terminal carbohydrate-recognition domain. Cterminal domain is responsible for lectin activity, while the 
presence of the N-terminal domain is necessary for full biological activity of galectin-3 $[9,10]$. It is found in a wide range of tissues [11]. Gal-3 lacks a secretion signal peptide for classical vesicle-mediated exocytosis, so it is localized primary not only in the cytoplasm but also in the nucleus of mitochondria. When secreted in the extracellular space Gal-3 can interact with cell surface receptors to initiate transmembrane signalling pathways for different cellular functions. Gal-3 is also necessary for normal macrophage function [12]. In granulocyte-macrophage colony-stimulating factor transgenic mice, the expression of Gal-3 was increased by $6-$ fold following the activation of macrophages [13]. Significant infiltration of macrophages was observed in hypertrophied heart, as well as in active myocarditis, and colocalized Gal3 was found $[1,14]$. Consequently, it can be concluded that Gal-3 has dependent stimulatory effect on macrophage migration. This hypothesis was confirmed by Sharma et al. by showing that exogenous recombinant Gal-3 significantly increased macrophage migration, an effect that was inhibited by the endogenous peptide $\mathrm{N}$-acetyl-seryl-aspartyllysyl-proline (Ac-SDKP) [15]. Henderson et al. found that macrophages had abundant Gal-3 within their nucleus and cytoplasm and that they were able to secrete substantial amounts of Gal-3 into the supernatant in the cell culture suggesting a location in the extracellular space [16]. In a murine model of hepatic fibrosis, Gal-3 was found to be localized by proliferating fibroblasts [17]. In normal rat cardiac fibroblasts, addition of exogenous recombinant Gal-3 significantly increased fibroblast proliferation, which resulted in an increased collagen production in hypertrophied heart [15]. Simultaneously, Gal-3 binding sites were localized around the nucleus of proliferating fibroblast, whereas resting cells only had minimal binding sites in the cytoplasm [18]. It was suggested that Gal-3 induced cardiac fibrosis via activation of cyclin D1, thus allowing a macrophage derived mediator to affect the myocardium [18]. Similarly, involvement of Gal-3 in development of fibrosis has been demonstrated not only in the heart [18] but also in the liver and in the kidney [16, 17].

Cardiac remodeling is a crucial element in the clinical outcome of heart failure, and therefore various drugs are administered to prevent ongoing damage to the heart. Sharma and colleagues showed that Gal-3 was the strongest differentially regulated gene by comparing compensated and decompensated left ventricular hypertension in rats [18, 19]. The assumption that galectin-3 was one of the causes involved in the onset of heart failure was corroborated by infusing Gal-3 into the pericardial sac of wild type rats, which prompted extensive myocardial fibrosis $[18,19]$. Other authors had similar results in murine model (angiotensin II-infused mice). Sharma et al. showed that coinfusion of Ac-SDKP along with galectin-3 into the pericardium may play a significant role in cardiac remodeling, not only in inhibiting fibrosis and inflammation but also in alleviating cardiac dysfunction [15].

Fibrosis and scar formation are a part of maladaptive mechanism to the injury. Fibroblasts and myofibroblasts as well as macrophages have been identified as key cells in development and growth of tissue scarring [20, 21]. Upregulation of Gal-3 was found in different human fibrotic conditions (liver cirrhosis, idiopathic lung fibrosis, and chronic pancreatitis) [17, 22-24]. Animal models of hepatic, renal, and cardiac fibrosis have also demonstrated the upregulation of Gal-3 [16, 17, 24]. Galectin-3 mRNA expression was significantly correlated with the extent of fibrosis. Inflammation is essential for tissue healing and scar formation. Sustained inflammation can lead to formation of extensive scar tissue and consequently to organ failure. Macrophages are a key cell type in development of fibrosis [25-27]. Specific depletion of macrophages significantly reduced myofibroblasts activation and decreased fibrosis (characterized by reduced alpha-SMA and collagen expression) [16].

The same authors demonstrated that disruption of Gal-3 gene did not affect macrophage recruitment or macrophage proinflammatory cytokine profiles in response to interferon $\gamma$ [16]. On the other hand, complete macrophage depletion in the Ren-2 rat model accelerated cardiac remodeling, supporting the notion that macrophages have a crucial role in remodelling [28]. We can conclude that galectin-3 and macrophages are major mechanisms in myofibroblast accumulation and activation, as well as in final fibrosis development.

\section{Galectin-3 and Heart Failure with Reduced Ejection Fraction}

The maladaptive changes that occur in surviving myocytes and in the extracellular matrix after myocardial injury lead to pathologic remodelling of the left ventricle, with dilatation and impaired contractility [29]. If these changes are left untreated, they worsen over time, exacerbated by additional injury and by systemic responses to left ventricular systolic dysfunction, notably activation of the sympathetic and reninangiotensin-aldosterone systems [30,31]. All these responses have detrimental systemic effects, accounting for the clinical manifestations of the syndrome of heart failure, including the development and worsening of symptoms, declining functional capacity, episodes of frank decompensation that result in the need for hospitalization, myocardial electrical instability, and premature death, usually due to pump failure or a ventricular arrhythmia [29]. Since the limited cardiac reserve of patients with systolic heart failure depends on atrial contraction and synchronized contraction of the left ventricle, events that affect these functions or that impose an additional hemodynamic load on the failing heart can lead to acute deterioration. Interruption of left ventricular remodeling and of the systemic responses to it is the basis of much of the effective treatment of heart failure [29].

The majorities of patients with HF have coronary artery disease and ischemic cardiomyopathy with zones of myocardial fibrosis as a respond to myocardial ischemia and infarction. Dilated cardiomyopathy may also result from a genetic cause, previous viral infection (recognized or unrecognized), alcohol abuse, or, occasionally, chemotherapy [29].

Increased stress and injury of the myocardium can contribute to myocardial remodeling [7]. Respond to myocardial damage involve recruitment of immune cells to the myocardium and production of signaling proteins from 
local pericytes, mast cells, and macrophages, resulting in activation of resident fibroblast and deposition of procollagen into extracellular matrix which leads to cardiac fibrosis. In the myocardium, aldosterone is a major stimulus for macrophages secretion of galectin-3, which in turn works as a paracrine signal on fibroblast to help translate the signal of transforming growth factor- $\beta$ to increase cyclin D1 and direct the proliferation of myofibroblast and collagen deposition [32]. Galectin-3 is the most upregulated protein in an animal model of left ventricular hypertrophy and HF [15]. Recombinant galectin-3 induced cardiac fibroblast proliferation, collagen production, and cyclin D1 expression. The same investigators demonstrated that intrapericardial infusion of galectin-3 into healthy rats increased left ventricle collagen density and reduced ejection fraction of left ventricle for $22 \%$. These data strongly suggest that galectin-3 is crucial for development of $\operatorname{HF}[15,33]$. Progressive cardiac fibrosis is central aspect of progressive systolic heart failure leading to creating tissue heterogeneity and stiffness that can cause sudden cardiac death by malignant arrhythmias $[29,32]$.

\section{Galectin-3 and Heart Failure with Preserved Ejection Fraction}

Diastolic dysfunction or heart failure with preserved ejection fraction (nonsystolic HF) represents an abnormality of diastolic distensibility, filling, or relaxation of the left ventricle, regardless of whether ejection fraction is normal or abnormal and whether the patients are with or without symptoms [30]. HFpEF has a different prognosis and treatment approach than HFrEF. Nonsystolic HF poses a challenge to diagnose with imaging modalities and the set of associated comorbidities such as advanced age, renal disorders, and diabetes [34]. It is known that angiotensin II directly and via stimulation of aldosterone is a crucial neurohormone involved in pathogenesis of cardiac fibrosis and impaired myocardial relaxation [34].

Zile et al. had demonstrated in one small series that galectin-3 levels were significantly elevated in cohort of patients with HFpEF [35]. Galectin-3 might provide an early warning marker for patients who are at risk for development of HF symptoms and may allow medical intervention. According to other animal and human studies, galectin-3 in addition to clinical and echocardiographic parameters can be used to confirm the presence of impaired diastolic function. Some studies have shown that galectin-3 had independent prognostic value, even after correction for established risk factor such as age, sex, BNP level, renal function, and diabetes mellitus [36]. Prognostic value of galectin-3 levels in plasma appears to be much stronger in the subset of patients with HFpEF in comparison with HFrEF [37]. Also, base line levels of galectin-3 seem to be sufficient to predict outcome, because serial measurement did not increase the prognostic yield [36].

\section{Galectin-3 as Biomarker}

Until recently, the goal of $\mathrm{HF}$ treatment was based on symptomatic relief. According to new trials and knowledge of myocardial remodelling as a crucial factor in HF development, slowing or reversing the progression of the disease is recognized as important goal of novel therapy (e.g., inhibition of angiotenzin-renin-aldosteron system) [36]. Identifying Gal-3 as an important segment in development of both myocardial remodelling and heart failure has opened possibility of Gal-3 being used as a new marker for the disease prognosis as well as a new treatment target.

Brain natriuretic peptide (BNP) and N-terminal probrain natriuretic peptide (NT-proBNP) assay were a golden standard in prognosis of $\mathrm{HF}$ in the past years. Natriuretic peptides are relisted by the myocardium as a result of myocardial stretching. In normal condition their level vary widley. In heart diseases condition, it is a marker of worsening heart failure and can present practical tool for heart failure treatment [37]. Troponin $\mathrm{T}$ and Troponin I (TnI/TnT) assay is useful in HF patients and according to trial data, slight elevations or chronically elevated levels of TnI/TnT predict a poor outcome [38]. Raised TnI/TnT levels are highly specific for myocyte injury. They are primarily used as markers in acute myocardial infarction, as well as in heart failure and some other conditions [39]. Like NTproBNP/BNP, the circulating TnI/TnT level is not pathogenic and specific and can be viewed as a signal of an ongoing pathological process in the heart. On the other hand, Gal-3 complements other HF biomarkers by providing an upstream signal of the myocardial fibrotic state, ventricular adverse remodelling, and progression of cardiomyopathy. Considering that cardiac fibrosis is irreversible process, Gal-3 measurement provides serological overview of the ongoing fibrotic process. BNP/NT-proBNP, TnT/TnI, and Gal-3 aid in prognosis, risk stratification, and management. Gal-3 levels are a direct reflection of cardiac fibrosis, are not acutely changed by HF decompensation, stay elevated once elevated in majority of cases, and are not affected by medical treatment [40]. DEAL-HF trial showed that plasma galectin-3 level has a prognostic value regardless of heart failure severity, as assessed by NT-proBNP levels, and it may be potentially used in management of such patients [41]. Galectin-3 and its prognostic value have been evaluated in number of studies. In 240 patients with stable chronic HF plasma Gal3 levels were strongly related to outcome [42]. In another trial, data for 599 patients presented with dyspnoea at the emergency department were analyzed by receiver operating characteristic analysis. The results showed that in two-month period mortality was higher in patients with higher plasma galectin-3 level, presenting with a greater area under the curve at 0.74 compared with NT-proBNP [37]. Multivariate logistic regression analysis revealed that elevated plasma galectin-3 level was the best independent predictor of 60day mortality or combination of death/recurrent HF within 60 days. Milting et al. found significantly elevated plasma galectin-3 levels at the time of mechanical circulatory support [43]. Patients who died had significantly higher plasma Gal3 level than those who were transplanted. This is also one more argument that galectin-3 plays an important role in myocardial remodelling and HF development. Galectin-3 is also associated with the risk of developing $\mathrm{HF}$ after acute coronary syndrome and supports potential clinical relevance 
of galectin-3-related pathway in patients with ischemic heart disease [44]. Galectin-3 was a strong independent predictor of 30-day major adverse cardiac outcome among patients with STEMI infarction undergoing primary PCI and thus can be utilized as a useful biomarker for stratifying high and low risk subgroups in daily clinical practice [45].

As mentioned before, various fibrotic conditions are associated with upregulation of galectin-3. Not only heart fibrosis but also upregulation of galectin-3 has been described in animal model for hepatic and renal fibrosis; in human liver cirrhosis; and in idiopathic lung fibrosis and chronic pancreatitis $[16,17,46-48]$. When taking into consideration importance of galectin-3 in heart failure pathophysiology, certainly we have to exclude the impact of these conditions on the final conclusions. Despite the fact that the frequent companion of heart failure is cardiorenal syndrome (renal failure), after correction for established risk factor (diabetes, age, sex, and renal function) it has been proven that high levels of galectin-3 has independent and significant impact on the prognosis of patients with heart failure $[36,49]$.

\section{Conclusion}

In every day clinical practice Gal-3 can be used to identify those patients at highest risk for readmission or death of HF. Galectin-3 measurement may be a significant factor in making a decision regarding visit intervals of whom to admit in patients with worsening HF. Due to the fact that Gal-3 levels are directly correlated with remodelling and fibrotic process in the myocardium, Gal-3 can be used as a culprit biomarker and can contribute to heart failure treatment as a potential novel target in therapeutics. This would be a real disease-modifying therapy to inhibit the remodelling process or slow down HF progression. Galectin-3 may one day allow identification and treatment of patients with coronary artery disease with a major risk of cardiomyopathy development.

\section{Conflict of Interests}

The authors declare that there is no conflict of interests regarding the publication of this paper.

\section{References}

[1] M. Gheorghiade and P. S. Pang, "Acute Heart Failure Syndromes," Journal of the American College of Cardiology, vol. 53, no. 7, pp. 557-573, 2009.

[2] R. S. Velagaleti and R. S. Vasan, "Epidemiology of heart failure," in Heart: A Companion to Braunwald's Heart Disease, D. L. Mann, Ed., pp. 346-354, Elsevier, Saint Louis, Mo, USA, 2nd edition, 2011.

[3] J. T. Thibodeau, A. T. Turer, S. K. Gualano et al., "Characterization of a novel symptom of advanced heart failure: bendopneas," JACC: Heart Failure, vol. 2, no. 1, pp. 24-31, 2014.

[4] K. Dickstein, A. Cohen-Solal, G. Filippatos et al., "ESC guidelines for the diagnosis and treatment of acute and chronic heart failure 2008: the Task Force for the diagnosis and treatment of acute and chronic heart failure 2008 of the European Society of Cardiology. Developed in collaboration with the Heart Failure
Association of the ESC (HFA) and endorsed by the European Society of Intensive Care Medicine (ESICM)," European Heart Journal, vol. 10, no. 10, pp. 933-989, 2008.

[5] J. J. McMurray, S. Adamopoulos, S. D. Anker et al., "ESC guidelines for diagnosis and treatment of acute and chronic heart failure 2012: the Task Force for the Diagnosis and Treatment of acute and chronic heart failure 2012 of the European Society of Cardiology. Developed in collaboration with the Heart Failure Association (HFA) of the ESC," European Journal of Heart Failure, vol. 14, pp. 803-869, 2012.

[6] S. A. Hunt, W. T. Abraham, M. H. Chin, and et al, "2009 Focused update incorporated into the ACC/AHA 2005 guidelines for the diagnosis and management of the heart failure in adults: a report of the AmericanCollege of Cardiology Foundation/American Heart Association task force on practice guidelines: developed in collaboration with the International Society for Heart and Lung Transplanation," Circulation, vol. 119, pp. e391-e479, 2009.

[7] V. V. Michels, P. P. Moll, F. A. Miller et al., "The frequency of familial dilated cardiomyopathy in a series of patients with idiopathic dilated cardiomyopathy," The New England Journal of Medicine, vol. 326, no. 2, pp. 77-82, 1992.

[8] E. E. Creemers and Y. M. Pinto, "Molecular mechanisms that control interstitial fibrosis in the pressure-overloaded heart," Cardiovascular Research, vol. 89, no. 2, pp. 265-272, 2011.

[9] J. Seetharaman, A. Kfanigsberg, R. Slaaby, H. Leffler, S. H. Barondes, and J. M. Rini, "X-ray crystal structure of the human galectin-3 carbohydrate recognition domain at 2.1resolution," The Journal of Biological Chemistry, vol. 273, no. 21, pp. 13047-13052, 1998.

[10] E. A. M. Barboni, S. Bawumia, K. Henrick, and R. C. Hughes, "Molecular modeling and mutagenesis studies of the $\mathrm{N}$ terminal domains of galectin-3: evidence for participation with the C-terminal carbohydrate recognition domain in oligosaccharide binding," Glycobiology, vol. 10, no. 11, pp. 1201-1208, 2000 .

[11] J. Dumic, S. Dabelic, and M. Flögel, "Galectin-3: an open-ended story," Biochimica et Biophysica Acta, vol. 1760, no. 4, pp. 616635, 2006.

[12] H. Sano, D. K. Hsu, J. R. Apgar et al., "Critical role of galectin3 in phagocytosis by macrophages," The Journal of Clinical Investigation, vol. 112, no. 3, pp. 389-397, 2003.

[13] M. J. Elliott, A. Strasser, and D. Metcalf, "Selective up-regulation of macrophage function in granulocyte-macrophage colonystimulating factor transgenic mice," The Journal of Immunology, vol. 147, no. 9, pp. 2957-2963, 1991.

[14] U. C. Sharma, S. Pokharel, T. J. van Brakel et al., "Galectin-3 marks activated macrophages in failure-prone hypertrophied hearts and contributes to cardiac dysfunction," Circulation, vol. 110, no. 19, pp. 3121-3128, 2004.

[15] U. Sharma, N.-E. Rhaleb, S. Pokharel et al., "Novel antiinflammatory mechanisms of N-Acetyl-Ser-Asp-Lys-Pro in hypertension-induced target organ damage," The American Journal of Physiology-Heart and Circulatory Physiology, vol. 294, no. 3, pp. H1226-H1232, 2008.

[16] N. C. Henderson, A. C. Mackinnon, S. L. Farnworth et al., "Galectin-3 expression and secretion links macrophages to the promotion of renal fibrosis," American Journal of Pathology, vol. 172, no. 2, pp. 288-298, 2008.

[17] N. C. Henderson, A. C. Mackinnon, S. L. Farnworth et al., "Galectin-3 regulates myofibroblast activation and hepatic 
fibrosis," Proceedings of the National Academy of Sciences of the United States of America, vol. 103, no. 13, pp. 5060-5065, 2006.

[18] K. Reifenberg, H.-A. Lehr, M. Torzewski et al., "Interferon$\gamma$ induces chronic active myocarditis and cardiomyopathy in transgenic mice," American Journal of Pathology, vol. 171, no. 2, pp. 463-472, 2007.

[19] B. Schroen, S. Heymans, U. Sharma et al., "Thrombospondin-2 is essential for myocardial matrix integrity: increased expression identifies failure-prone cardiac hypertrophy," Circulation Research, vol. 95, no. 5, pp. 515-522, 2004.

[20] S. L. Friedman, "Molecular regulations of hepatic fibrosis, an integrated cellular response to tissue injury," The Journal of Biological Chemistry, vol. 275, no. 4, pp. 2247-2250, 2000.

[21] J. S. Duffield, S. J. Forbes, C. M. Constandinou et al., "Selective depletion of macrophages reveals distinct, opposing roles during liver injury and repair," Journal of Clinical Investigation, vol. 115, no. 1, pp. 56-65, 2005.

[22] D. K. Hsu, C. A. Dowling, K. C. Jeng, J. T. Chen, R. Y. Yang, and F. T. Liu, "Galectin-3 expression is induced in cirrhotic liver and hepatocellular carcinoma," International Journal of Cancer, vol. 81, no. 4, pp. 519-526, 1999.

[23] Y. Nishi, H. Sano, T. Kawashima et al., "Role of galectin-3 in human pulmonary fibrosis," Allergology International, vol. 56, no. 1, pp. 57-65, 2007.

[24] S. Sasaki, Q. Bao, and R. C. Hughes, "Galectin-3 modualtes rat mesengial cell proliferation and matrix synthesis during experimental glomerulonephritis induced by anti-Thyl.1 antibodies," The Journal of Pathology, vol. 187, pp. 481-489, 1999.

[25] H.-J. Anders, V. Vielhauer, M. Frink et al., "A chemokine receptor CCR-1 antagonist reduces renal fibrosis after unilateral ureter ligation," The Journal of Clinical Investigation, vol. 109, no. 2, pp. 251-259, 2002.

[26] V. Eis, B. Luckow, V. Vielhauer et al., "Chemokine receptor CCR1 but nor CCR5 mediates leukocyte recruitment and subsequent renal fibrosis after unilateral obstruction," Journal of the American Society of Nephrology, vol. 15, no. 2, pp. 337-347, 2004.

[27] K. Kitagawa, T. Wada, K. Furuichi et al., "Blockade of CCR2 ameliorates progressive fibrosis in kidney," The American Journal of Pathology, vol. 165, no. 1, pp. 237-246, 2004.

[28] H. R. Zandbergen, U. C. Sharma, S. Gupta et al., "Macrophage depletion in hypertensive rats accelerates development of cardiomyopathy," Journal of Cardiovascular Pharmacology and Therapeutics, vol. 14, no. 1, pp. 68-75, 2009.

[29] J. J. V. McMurray, "Systolic heart failure," The New England Journal of Medicine, vol. 362, no. 3, pp. 228-238, 2010.

[30] W. H. Gaasch and M. R. Zile, "Left ventricular diastolic dysfunction and diastolic heart failure," Annual Review of Medicine, vol. 55, pp. 373-394, 2004.

[31] P. Abrahamsson, J. Dobson, C. B. Granger et al., "Impact of hospitalization for acute coronary events on subsequent mortality in patients with chronic heart failure," European Heart Journal, vol. 30, no. 3, pp. 338-345, 2009.

[32] G. P. Aurigemma and W. H. Gaasch, "Diastolic heart failure," The New England Journal of Medicine, vol. 351, no. 11, pp. 10971157, 2004.

[33] Y.-H. Liu, M. D’Ambrosio, T. D. Liao et al., "N-acetylseryl-aspartyl-lysyl-proline prevents cardiac remodeling and dysfunction induced by galectin-3, a mammalian adhesion/growth-regulatory lectin," The American Journal of Physiology-Heart and Circulatory Physiology, vol. 296, no. 2, pp. H404-H412, 2009.
[34] P. A. McCullough, A. Olobatoke, and T. E. Vanhecke, "Galectin3: a novel blood test for the evaluation and management of patients with heart failure," Reviews in Cardiovascular Medicine, vol. 12, no. 4, pp. 200-210, 2011.

[35] M. R. Zile, S. M. De Santis, C. F. Baicu et al., "Plasma galectin3 levels in patinets with structural and clinical manifestation of hypertensive heart disease: relationship to determination of matrix composition," Circulation, vol. 122, Article ID A12433, 2010.

[36] R. A. de Boer, A. A. Voors, P. Muntendam, W. H. van Gilst, and D. J. van Veldhuisen, "Galectin-3: a novel mediator of heart failure development and progression," European Journal of Heart Failure, vol. 11, no. 9, pp. 811-817, 2009.

[37] G. Savarese, B. Trimarco, S. Dellegrottaglie et al., "Natriuretic peptide-guided therapy in chronic heart failure: a meta-analysis of 2,686 patients in 12 randomized trials," PLoS ONE, vol. 8, no. 3, Article ID e58287, 2013.

[38] R. D. Kociol, P. S. Pang, M. Gheorghiade, G. C. Fonarow, C. M. O'Connor, and G. M. Felker, "Troponin elevation in heart failure: prevalence, mechanisms, and clinical implications," Journal of the American College of Cardiology, vol. 56, no. 14, pp. 1071-1078, 2010.

[39] A. Tanindi and M. Cemri, "Troponin elevation in conditions other than acute coronary syndromes," Vascular Health and Risk Management, vol. 7, no. 1, pp. 597-603, 2011.

[40] D. J. A. Lok, P. van der Meer, P. W. B.-A. de la Porte et al., "Prognostic value of galectin-3, a novel marker of fibrosis, in patients with chronic heart failure: data from the DEAL-HF study," Clinical Research in Cardiology, vol. 99, no. 5, pp. 323328, 2010.

[41] D. Lok, P. van der Meer, P. B. de la Porte et al., "Galectin3, a novel marker of macrophage activity, predicts outcome in patients with stabile chronic heart failure," Journal of the American College of Cardiology A, vol. 49, article 98, 2007, (abstract).

[42] R. R. van Kimmenade, J. L. Januzzi Jr., P. T. Ellinor et al., "Utility of amino-terminal pro-brain natriuretic peptide, galectin-3, and apelin for the evaluation of patients with acute heart failure," Journal of the American College of Cardiology, vol. 48, no. 6, pp. 1217-1224, 2006.

[43] H. Milting, P. Ellinghaus, M. Seewald et al., "Plasma biomarkers of myocardial fibrosis and remodelling in terminal heart failure patients supported by mechanical circulatory support devices," Journal of Heart and Lung Transplantation, vol. 27, no. 6, pp. 589-596, 2008.

[44] E. W. Grandin, P. Jarolim, S. A. Murphy et al., "Galectin-3 and the development of heart failure after acute coronary syndrome: pilot experience from PROVE IT-TIMI 22," Clinical Chemistry, vol. 58, no. 1, pp. 267-273, 2012.

[45] T.-H. Tsai, P.-H. Sung, L.-T. Chang et al., "Value and level of galectin-3 in acute myocardial infarction patients undergoing primary percutaneous coronary intervention," Journal of Atherosclerosis and Thrombosis, vol. 19, no. 12, pp. 1073-1082, 2012.

[46] L. Wang, H. Friess, Z. Zhu et al., "Galectin-1 and galectin-3 in chronic pancreatitis," Laboratory Investigation, vol. 80, no. 8, pp. 1233-1241, 2000.

[47] D. K. Hsu, C. A. Dowling, K. C. Jeng, J. T. Chen, R. Y. Yang, and F. T. Liu, "Galectin-3 expression is induced in cirrhotic liver and Hepatocellular carcinoma," International Journal of Cancer, vol. 81, no. 4, pp. 519-526, 1999. 
[48] Y. Nishi, H. Sano, T. Kawashima et al., "Role of galectin-3 in human pulmonary fibrosis," Allergology International, vol. 56, no. 1, pp. 57-65, 2007.

[49] R. A. De Boer, D. J. A. Lok, T. Jaarsma et al., "Predictive value of plasma galectin-3 levels in heart failure with reduced and preserved ejection fraction," Annals of Medicine, vol. 43, no. 1 , pp. 60-68, 2011. 


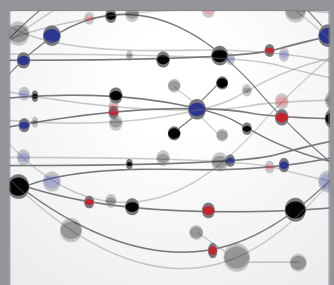

The Scientific World Journal
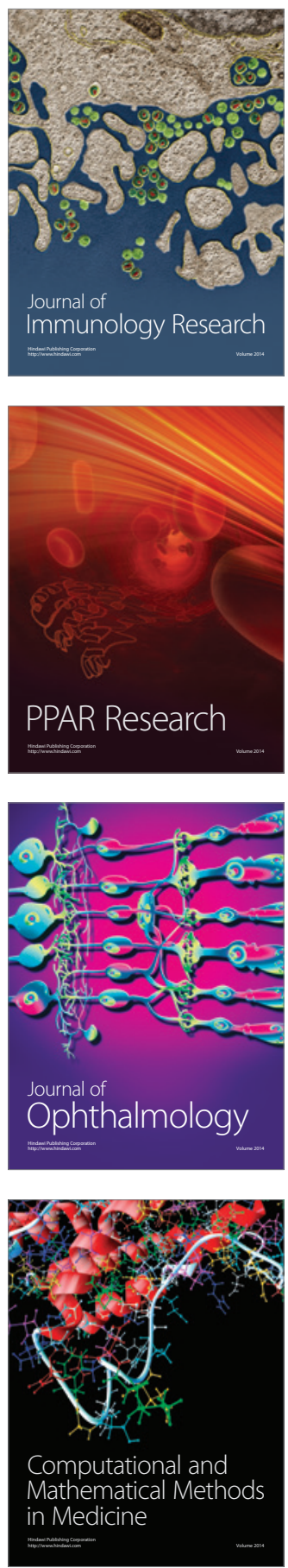



Gastroenterology

Research and Practice
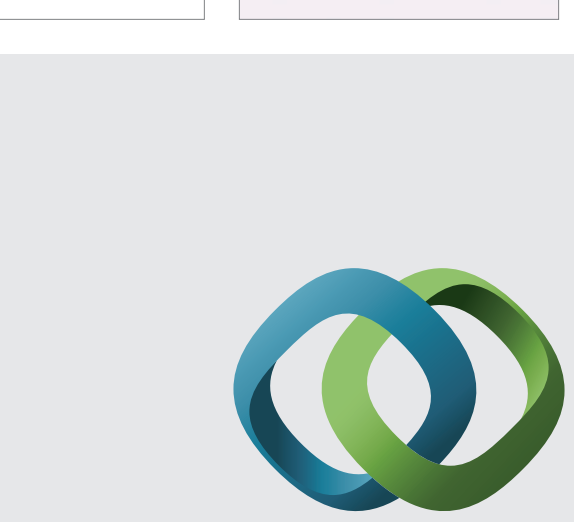

\section{Hindawi}

Submit your manuscripts at

http://www.hindawi.com

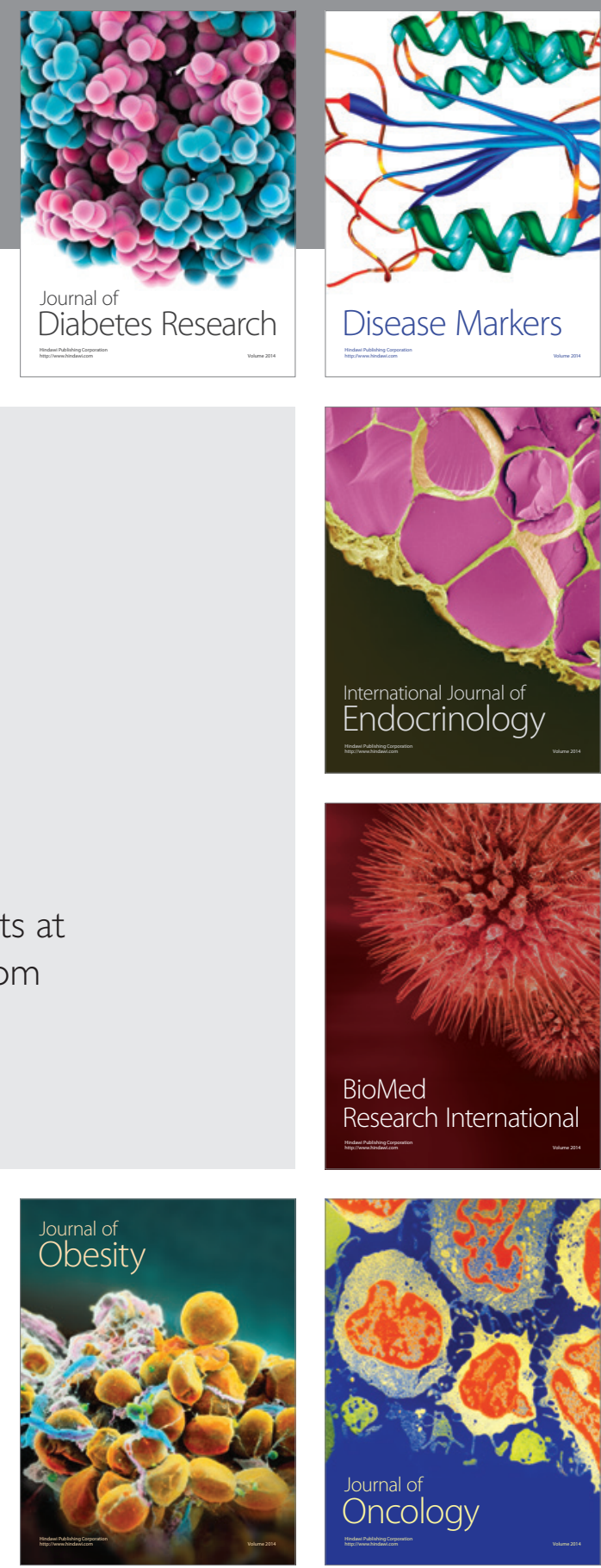

Disease Markers
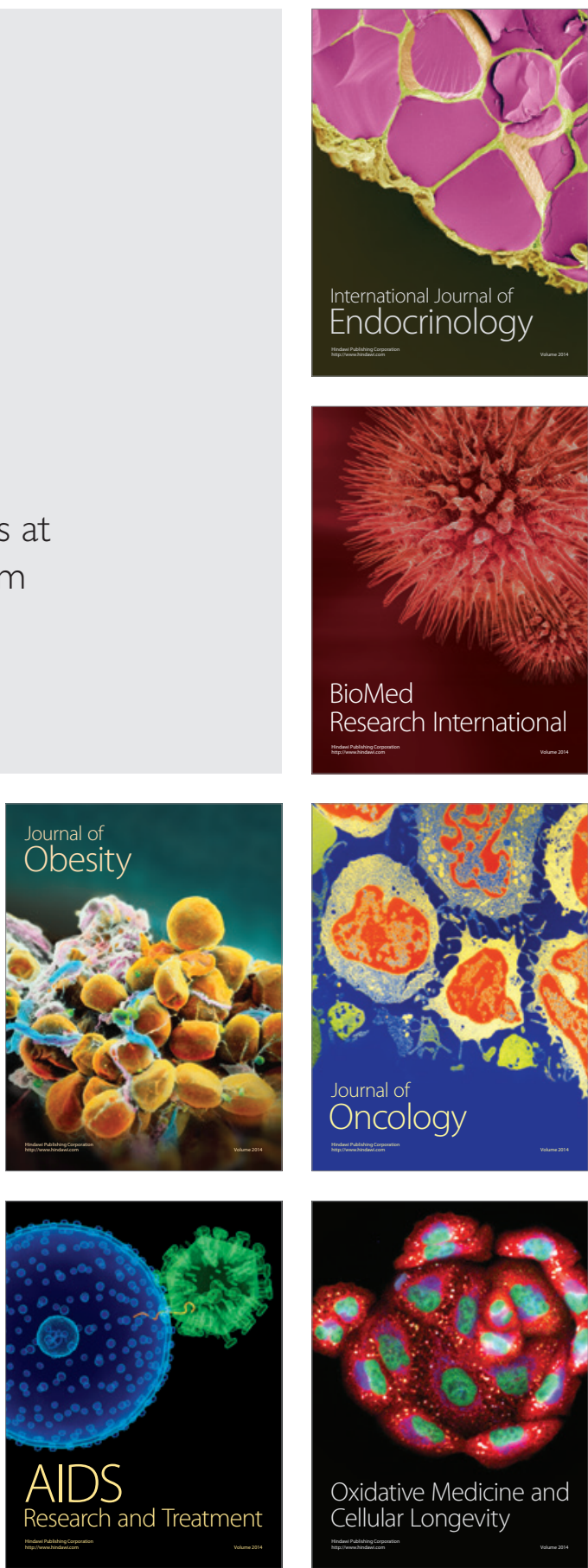